\title{
Multiphase flow metering with nuclear magnetic resonance spectroscopy
}

Attila M. Bilgic, KROHNE Messtechnik GmbH, Duisburg, Germany, a.bilgic@krohne.com

Johannes W. Kunze, KROHNE Messtechnik GmbH, Duisburg, Germany

Volker Stegemann, KROHNE Messtechnik GmbH, Duisburg, Germany

Jankees Hogendoorn, KROHNE New Technolgies, Dordrecht, Netherlands

Marco Zoeteweij, KROHNE New Technolgies, Dordrecht, Netherlands

\section{Kurzfassung}

Die Messung von Fluiden in der Öl- und Gasindustrie erfordert das zuverlässige Messen von Mehrphasenströmungen. Für die Aufgabe verspricht Magnetische Resonanz viele Vorteile gegenüber bisher eingesetzten Technologien. Dieser Beitrag beschäftigt sich mit einem neu entwickelten Durchflussmessgerät, das auf magnetischer Resonanz basiert und die Zusammensetzung von Mehrphasenströmungen ermöglicht. Dazu werden zunächst die Grundlagen der magnetischen Resonanz beschrieben. Danach werden die Details der technischen Umsetzung und das Verfahren zur Bestimmung der der Zusammensetzung von Strömen erläutert.

\begin{abstract}
The measurement of fluids in the oil and gas industry requires a robust measurement of multiphase flows. Magnetic resonance as a measurement principle has multiple advantages over existing technologies. A magnetic resonance based multiphase flow meter which is capable of producing an image of the spatial distribution of a mulitphase flow has been developed. This paper describes the principles of magnetic resonance. Afterwards details of the technical implementation and the method by which the system determines multiphase flow composition are explained.
\end{abstract}

\section{Introduction}

Multiphase and wet gas flow meters provide essential online and continuous flow rate information in upstream oil and gas production systems. During the last years, the associated technology has been matured such that acceptable performance can be achieved over a range of flow conditions. Nevertheless, a number of limitations persist and several specific requirements have not been adequately addressed by existing technologies. Additionally existing technology has drawbacks such as the use of radioactive sources, the utilization of differential pressure measurement, or the need of a priori knowlegde of fluid properties at line conditions. During the last decade magnetic resonance technology has matured and is being used for various applications.

For many years, magnetic resonance based logging tools have been successfully used for detailed formation evaluation [1]. Magnetic resonance has specific advantages that can also be used for multiphase flow measurement [2], [3]. This paper describes the principles of a magnetic resonance flow meter in the field of multiphase flow measurement in the oil and gas industry.

\section{Magnetic Resonance Principle}

\subsection{Precession and Resonance Frequency}

Magnetic resonance utilizes the response of nuclei located in a magnetic field to Radio Frequency (RF) pulses. As a consequence atoms without a magnetic moment are invisible to MR. Magnetic moment in nuclei is carried by neutrons and protons. Therefore only atoms with an odd number of protons, neutrons, or both protons and neutrons will have an effective magnetic moment. Such nucleis are for example ${ }^{1} \mathrm{H},{ }^{13} \mathrm{C}$, or ${ }^{2} \mathrm{H}$. Only atoms with a sufficiently high natural abundance can be utilized in MR experiments. Typically hydrogen is targeted by oil-field applications [4], [5]. This is due to the strong response of the ${ }^{1} \mathrm{H}$ nucleus in MR experiments as well as the high amount of hydrogen in such applications. Normal hydrogen contains almost exclusively ${ }^{1} \mathrm{H}$ nuclei. Water $\left(\mathrm{H}_{2} \mathrm{O}\right)$ as well as hydrocarbons, which can be found in crude oil, contain high amounts of hydrogen. When exposed to an external magnetic field $\left(\vec{B}_{0}\right)$ the nuclei align themselves either with or against the direction of the external field. This is due to the torque that the external magnetic field exerts on the axis of the spinning magnetic moments. Another result is a movement perpendicular to the direction of the applied torque and thus a precession around the direction of the external magnetic field. This motion is called Larmor precession. The frequency with which the nuclei precess around the axis is referred to as the Larmor frequency. This frequency can be derived via the Vector of the Magnetic field and the gyromegnetic ration $\gamma$ :

$$
f_{0}=-\frac{\gamma}{2 \pi}\left|\vec{B}_{0}\right|
$$

The gyromagnetic ratio is a material constant. Typically the strength of the used permanent magnets is chosen as such that precess frequencies fall into the range of several hunders of $\mathrm{kHz}$ to a few $\mathrm{MHz}$.

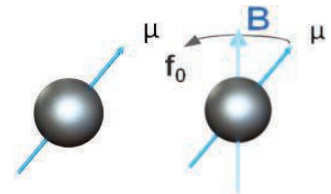


Figure 1: Two ${ }^{1} \mathrm{H}$ protons. Left-hand figure: no external static magnetic field. Right-hand figure: with external static magnetic field; the ${ }^{1} \mathrm{H}$ proton starts to precess along the magnetic field direction with the resonance (Larmor) frequency $\mathrm{f}_{0}$.

\subsection{Magnetization}

The direction and magnitude of the angular momentum of a nucleus is quantized in multiples of $\hbar$ (Planck's constant, $h$, divided by $2 \pi$ ). Due to this, nuclei always align themselves in distinct angles relative to a magnetic field.

In case of ${ }^{1} \mathrm{H}$ nuclei, only two distinct alignments in respect to the external magnetic field are possible. ${ }^{1} \mathrm{H}$ nuclei can be aligned at a certain angle either with or against the magnetic field. The distribution into these two states is not equal. The population of these states can be approximated by the ratio of magnetic to thermal energy. At room temperature the position aligned with the magnetic field has a lower energy state and is in consequence slightly favored. Within one million nuclei there is about one nucleus aligned with the magnetic field. On the macroscopic level this creates a magnetization $\vec{M}$ that is pointing in the direction of the external magnetic field.

This magnetization $\vec{M}$ builds up over time. For a simple compound consisting of one fluid this build-up can be described by an exponential function with one time constant called longitudinal relaxation time. The magnetization over time can be seen in Figure 2. A more complex fluid or a mixture of mutliple fluids will typically be characterized by a spectrum of longitudinal relaxation times.

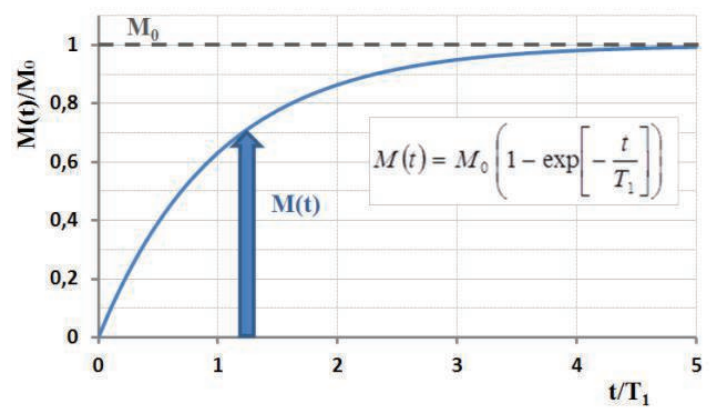

Figure 2: Time-dependent magnetization build-up. The longitudinal relaxation time, $\mathrm{T}_{1}$, characterize the build-up of the magnetization for a single-phase fluid.

\subsection{Radio Frequency Pulses}

By transmitting radio-frequency (RF) pulses from an antenna to the fluid, the alignment of the magnetic moments can be influenced. When a pulse with the appropriate intensity, duration, and frequency is applied, the orientation and intensity of the macroscopic magnetization $\vec{M}$ is therefore changed. With an appropriate RF coil the change can be measured as a voltage. This voltage is referred to as the MR signal in magnetic resonance experiments. The effect is called Hahn-Echo or spin Echo.

\subsubsection{Hahn-Echo}

The Spin-Echo was discovered in an experiment by Erwin Hahn in 1950. Further development has been done by Carr and Purcell. To create a spin echo, an RF pulse is first created with such an intensity and duration that the magnetization vector $\vec{M}$ is rotating with the Larmor frequency in the xy-plane. This is achieved by tilting the vector by $90^{\circ}$ from the z-orientation. The pulse is thus called a P90 pulse (Figure 3). Spatial inhomogeneity in the magnetic field strength leads to slightly differing Larmor frequencies of protons located in different positions. In regions with lower local field strength protons slow down whereas protons in regions with higher local field strength speed up in their larmor precession. This is illustrated in Figure $3 b$.

As a consequence, the phase cohenrence slowly disappears, which, in turn, diminishes the magnetization vector and leads to a fast decaying signal.

To counteract this a P180 pulse is applied that flips all protons on the y-axis. This puts the slower moving protons ahead of the faster moving protons. Since the individual resonance frequency is unchanged, all protons start to rephase (Figure $3 \mathrm{c}$ ), leading to an echo at $t=2 \tau$ (Figure 3d). This echo is called the Hahn echo.

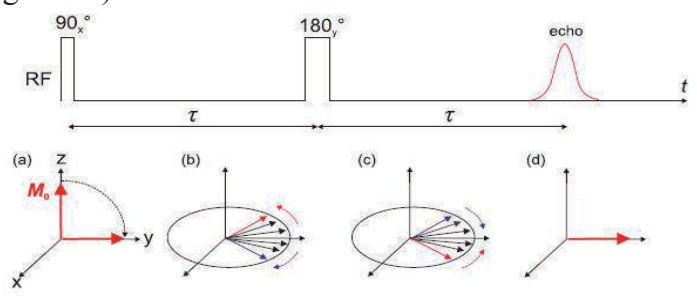

Figure 3: The Hahn-experiment with a) P90 pulse b) dephasing signal mainly due to differences in the local magnetic field, c) re-phasing signal after $\mathrm{P} 180$ pulse at $\mathrm{t}=\tau$, d) echo at $t=2 \tau$.

\subsubsection{CPMG Pulse Sequence}

By repeatedly applying P180 pulses, spin echoes can be created multiple times. This pulse sequence is called the CPMG pulse sequence and is illustrated in Figure 6. The sequence is named after its inventors: Carr, Purcell, Meiboom and Gill [5]. The sequence can be modified by varying the time delay, $\mathrm{T}_{\mathrm{E}}$, between subsequent $\mathrm{P} 180$ pulses. Different physical and chemical processes can be studied this way. Inter-echo spacings between tens or hundreds of microseconds to several milliseconds are typically applied during the CPMG sequence. Several thousand echoes can be measured in one run.

During a CPMG sequence, the amplitudes of succeeding echoes are slightly attenuated relative to the previous echo because the reversal of the direction of the Larmor precession by a P180 pulse does not perfectly compensate for differences in the Larmor frequencies of different spins. Therefore, the signal envelope of all echoes acquired during the CPMG sequence eventually decays to noise level. This signal decay describes the return of the 
magnetic moments to their equilibrium state prior to the disturbance by RF pulses and is characterized by the socalled transverse relaxation time, $\mathrm{T}_{2}$. This is shown in Figure 5. In weak, homogenous magnetic fields, and for simple, symmetric, molecules, the $\mathrm{T}_{1}$ - and $\mathrm{T}_{2}$-relaxation times of fluids are similar, since both are governed by the molecular structure of the fluid (such as inter- and intramolecular distances between the hydrogen atoms) as well as the ratio of viscosity to temperature [6].
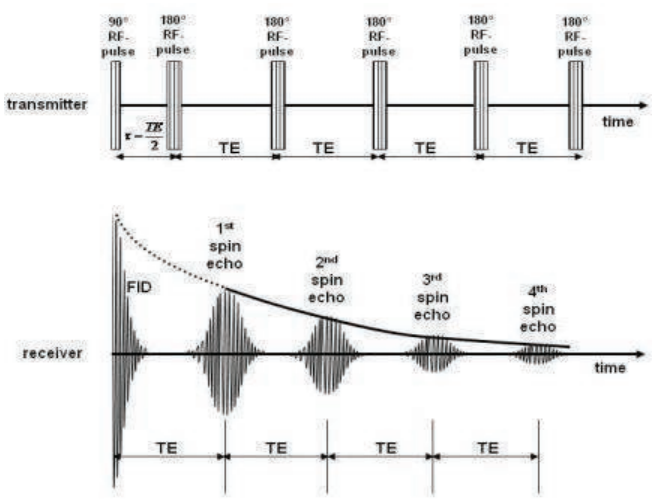

Figure 4: Illustration of the CPMG pulse sequence with inter-echo spacing of TE, creating multiple echoes.

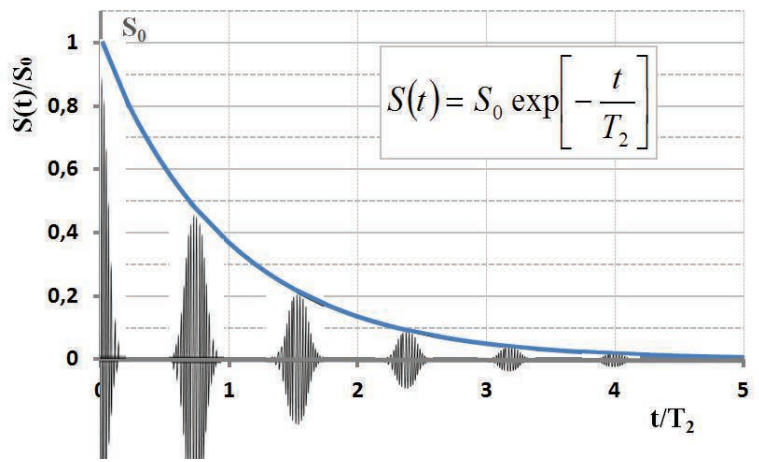

Figure 5: The decay of the spin echoes is characterized by the transverse relaxation time $\mathrm{T}_{2}$

Besides CPMG, a variety of RF pulse sequences have been developed for measuring $T_{1}$ or $T_{2}$. By varying the timing of these pulse sequences, the measurement can be optimized to the expected MR response of the flowing fluids.

\section{MR for Multiphase Flow Meas- urement}

The magnetic resonance multiphase flow meter uses the concepts which are described in the previous chapter. It consists of (Figure 6)

- a pre-magnetization section and

- a perturbation and measurement section involving a radio-frequency coil.
Within the pre-magnetization section the magnetic moments of hydrogen atoms are aligned by application of a constant magnetic field $\mathrm{B}_{0}$.

In the measurement section electromagnetic radio frequency (RF) pulses are generated which perturb the alignment of hydrogen atoms. The hydrogen atoms emit $\mathrm{RF}$ pulses during their return to equilibrium alignment in the external magnetic field which are detected and evaluated.

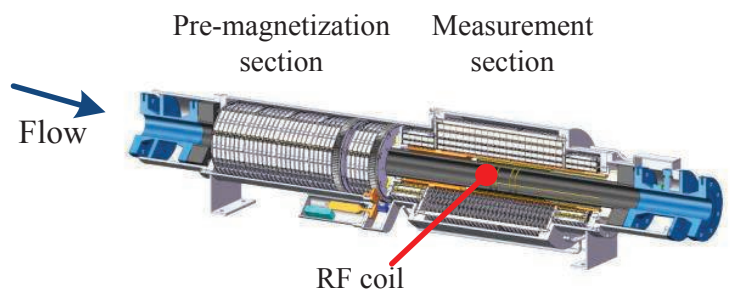

Figure 6: Simplified drawing of the magnetic resonance multiphase flow meter.

\subsection{Determination of Water Liquid Ratios}

In the pre-magnetization section, the hydrogen atoms are magnetized and a net macroscopic magnetization is created. This process is depicted in Figure 2 as function of time. In the flow meter, magnetization as function time can be transformed to magnetization as function of distance to the start of the pre-magnetization section by substituting the simple relation $t=x / v$ where $x$ is the position within the pre-magnetization section and $v$ is the flow velocity. The magnetization as function of $x$ for a specific velocity and longitudinal relaxation time, $\mathrm{T}_{1}$, is shown in Figure 7.

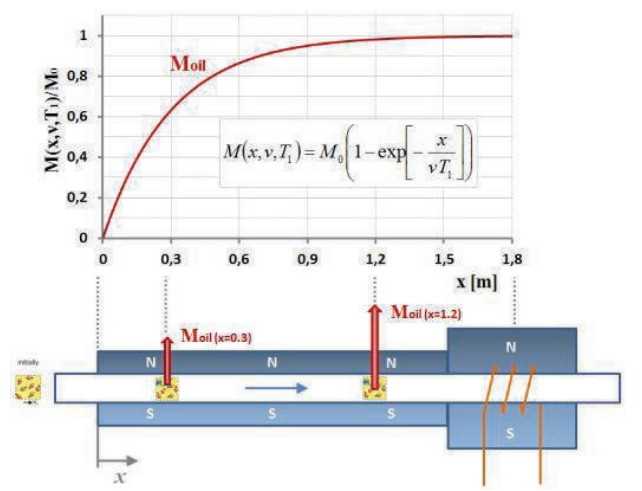

Figure 7: Magnetization build-up as a function of position inside the pre-magnetization section for a specific velocity and $\mathrm{T}_{1} .\left(\mathrm{v}=2[\mathrm{~m} / \mathrm{s}], \mathrm{T}_{1 \text { oil }}=0.15 \mathrm{~s}\right)$.

An important property of oil and water, which is exploited in the MR multiphase flow meter, is the difference in longitudinal relaxation time, $\mathrm{T}_{1}$. For most oil viscosities encountered in the industry, the longitudinal relaxation time, $T_{1}$, is significantly shorter than that of water. As a consequence, oil magnetizes faster than water (Figure 8, left-hand figure). This difference in the rate of magnetization build-up is used to create contrast between oil and water. By varying the effective length of the pre- 
magnetization section, the signals originating from oil and water, respectively, will be built-up to different levels, leading to quantitative information on the water-oil ratio. This process is illustrated in Figure 8.

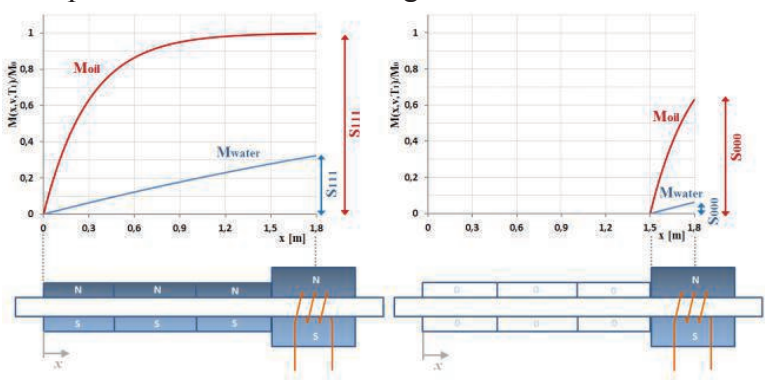

Figure 8: Build-up of magnetization and, hence, signal levels, for oil and water achieved with maximum premagnetization length (left-hand figure) and minimum premagnetization length (right-hand figure). The magnetization build-up has been calculated for a flow velocity, $\mathrm{v}=$ $2 \mathrm{~m} / \mathrm{s}$ and assuming longitudinal relaxation times, T1;oil $=$ $0.15 \mathrm{~s}$ and $\mathrm{T}_{1}$;water $=2 \mathrm{~s}$.

In the left-hand figure, the full pre-magnetization length (labeled as configuration 111) is used. For the particular flow velocity displayed, this length is sufficient to achieve complete polarization of the oil signal, whereas the signal originating from water has been built up to approximately $30 \%$. Consequently, the oil-water signal ratio $\left(\mathrm{S}_{11 \text { loil }} / \mathrm{S}_{11 \text { water }}\right)$ in the measuring section adjacent to the pre-magnetization section is about 3 . When the premagnetization length is reduced to just the length of the measuring section magnet (labeled as configuration 000), both the oil- and water signals are reduced but the signal from water is more strongly attenuated than the oil signal. This leads to an oil-water signal ratio $\left(\mathrm{S}_{0000 \mathrm{il}} / \mathrm{S}_{000 \text { water }}\right)$ of about 10 (right-hand side).

Figure 10 demonstrates that a strong contrast can be created between the signals originating from oil and from water based on the difference in $\mathrm{T}_{1}$ times by varying the pre-magnetization length. This contrast enables precise quantification of the oil-water ratio even for very high water liquid ratios. This capability is illustrated in Figure 9. In this figure, the signal ratio $\mathrm{S}_{000} / \mathrm{S}_{111}$ of both oil (red) and water (blue) are plotted as a function of flow velocity.

s000/S111 weighted with signal amplitude (SNR taken into account)

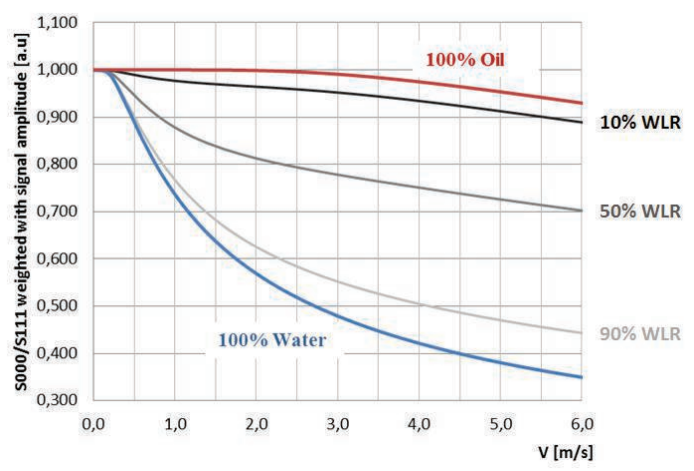

Figure 9: Signal ratio $\mathrm{S}_{000} / \mathrm{S}_{111}$ as function of flow velocity for various oil-water ratios. The signal intensities have been calculated assuming longitudinal relaxation times of
$0.15 \mathrm{~s}$ for oil and $2.3 \mathrm{~s}$ for water. A strong signal contrast between oil and water can be achieved even for high flow rates.

For multiphase flow of oil and water, the $\mathrm{S}_{000} / \mathrm{S}_{111}$ curve will always fall between the pure oil and pure water curves. The shape of curves for different water liquid ratios can be calculated and has been added to Figure 9 for water liquid ratios of $10 \%, 50 \%$ and $90 \%$, respectively. Figure 9 also shows that the sensitivity of the MR flow meter for determining the water liquid ratio (WLR) increases with increasing water liquid ratio, demonstrated by an increased separation of the water liquid ratio lines with increasing water liquid ratio. This method remains robust even when phase slip between the oil and water phases is present.

The water liquid ratio determination method is largely independent of the gas volume fraction (GVF) up to high GVF's because the gas signal is weak compared to the liquid signal. Once the gas fraction has been determined, as discussed in the next paragraph, the gas signal can be subtracted from the respective signals $\mathrm{S}_{000}$ and $\mathrm{S}_{111}$. This process produces liquid-only MR signals, which are used for water liquid ratio determination

\subsection{Determination of Gas Hold-Up}

The MR flow meter is capable of directly measuring the gas hold-up as well as the gas phase velocity. The underlying principles will be explained in this and subsequent paragraphs. Assume a simplified two phase flow situation comprising a gas-liquid mixture.

For this system holds that the sum of the liquid and gas hold-ups equals unity:

$$
\lambda_{L}+\lambda_{G}=1
$$

where $\lambda_{\mathrm{L}}$ and $\lambda_{\mathrm{G}}$ are the hold-ups for liquid and gas, respectively.

Furthermore, we know that the measured signal, $\mathrm{S}_{\text {meas. }}$, is a superposition of the signals generated by the liquid and gas fractions, respectively:

$$
\lambda_{L} \cdot S_{10 \% \mathrm{~L}}+\lambda_{G} \cdot S_{10 \% \mathrm{G}}=S_{\text {meas. }}
$$

where $\mathrm{S}_{100 \% \mathrm{~L}}$ and $\mathrm{S}_{100 \% \mathrm{G}}$ are the signal amplitudes corresponding to $100 \%$ liquid and gas filling, respectively. These particular-signals amplitudes are determined as part of the calibration procedure for each meter.

Equation (1) in combination with (2) can be written as:

$$
\lambda_{G}=\frac{S_{10 \% L .}-S_{\text {meas. }}}{S_{10 \% \text { L }}-S_{10 \% \% G}}
$$

$\mathrm{S}_{\text {meas. }}, \mathrm{S}_{100 \% \mathrm{~L}}$ and $\mathrm{S}_{100 \% \mathrm{G}}$ are known. Consequently, $\lambda_{\mathrm{G}}$ can be calculated. Considering the strong contrast between the liquid and gas signal amplitudes due to their contrast in hydrogen density, this methodology is a very robust and reliable way to accurately determine the gas hold-up. This approach can be extended for the situation in which the liquid phase is a composition of oil and water. 


\subsection{Determination of Flow Velocity}

The flow velocity is determined by using the so-called convective decay method. In the measurement section, the alignment of hydrogen atoms relative to the direction of the external magnetic field is perturbed by irradiating RF energy using an RF coil and a pre-defined pulse sequence, such as a CPMG sequence introduced earlier. The spin echoes produced by the sample are detected by the same RF coil also used for irradiating the RF energy. The amplitude of the initial Hahn echo has the highest value since all excited protons are still inside the RF coil. In contrast, when the second Hahn echo is formed about $1 \mathrm{~ms}$ later, a certain fraction of excited protons has already flown out of the RF coil. Consequently, the amplitude of the second echo will be somewhat lower than that of the first echo, even if there was no transverse relaxation. All following echoes are successively attenuated, since more and more protons that were excited by the initial RF pulse have left the RF coil. This 'convective decay' of the echo signals is shown in Figure 10.

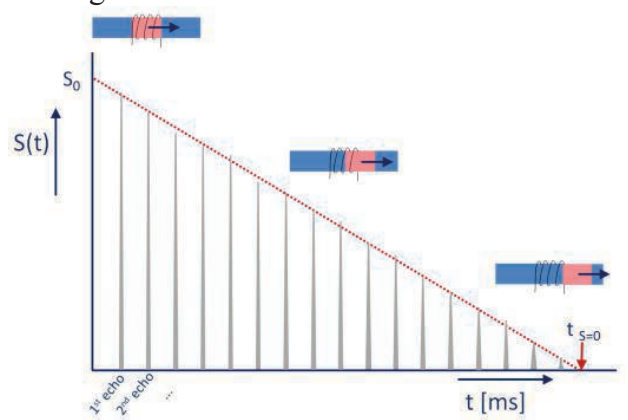

Figure 10 Velocity determination using the so-called convective decay method. Ignoring magnetic relaxation effects, the measured echo amplitude decreases linearly with time since the excited protons are leaving the RF coil due the flow. At a certain time all excited protons have left the RF coil. The RF coil length divided by the time at which the red dashed line intercepts the horizontal axis yields flow velocity.

The signal decay is proportional to the flow velocity. The higher the flow the faster the echo decay. The flow velocity is equal to the length of the RF coil, $\mathrm{L}_{c}$ (typically $10 \mathrm{~cm}$ ), divided by the time at which the red dashed curve intercepts the $\mathrm{x}$-axis, $\mathrm{t}_{\mathrm{S}=0}$, so $\mathrm{v}=\mathrm{L}_{\mathrm{c}} / \mathrm{t}_{\mathrm{S}=0}[7]$.

Combining the convective decay method with varying the pre-magnetization length enables the direct measurement of velocity slip between the flowing liquid phases. As shown in Figure 8, signal contrast can be created between water and oil by modifying the effective premagnetization length. For a long pre-magnetization length, the initial signal, $\mathrm{S}_{0}$, is a composition of signal contributions originating from both oil and water (Figure 10).

The velocity that is obtained from the convective signal decay is a composition of the oil and water velocities. In contrast to this, the initial signal amplitude corresponds predominantly to oil if a short pre-magnetization length is selected, cf. Figure 10. Consequently, the velocity that is being measured for this pre-magnetization configuration predominantly reflects the oil velocity. This, in combination with the measured water liquid ratio, makes it possible to determine both the oil and water velocities independently.

\subsection{Determination of Flow Composition and Velocity Profile}

Similar to Magnetic Resonance Imagers developed for medical applications, the MR multiphase flow meter is capable of producing an image of the spatial distribution of hydrogen protons inside the pipe.

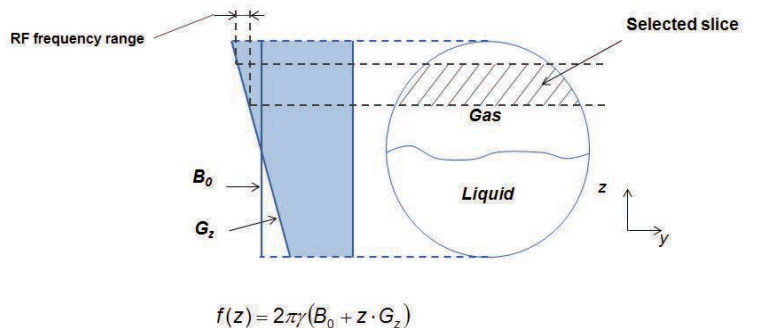

Figure 11 Application of a linear magnetic field gradient in the vertical direction, $\mathrm{z}$, makes the proton resonance frequency to become a function of height inside the pipe. By means of an RF frequency band selection, only protons in the selected slice can be measured. It should be noted that the flow is in the $\mathrm{x}$-direction.

The resonance frequency of detecting a MR signal is directly proportional to the strength of the applied magnetic field, $\mathrm{B}_{0}$.

By adding a linear magnetic field gradient, $G_{z}$, in the vertical direction, see Figure 11, the magnetic field strength varies linearly inside the pipe and now becomes a function of height, as indicated by the blue shaded area. Consequently, the resonance frequency of the protons, $f$, becomes a function of the height inside the pipe, $\mathrm{z}$.

By means of an RF frequency band selection, only protons in the corresponding resonance frequency band are read out, effectively leading to a slice-selective measurement. The approach to determine the WLR, gas hold-up and velocity, as just discussed, could be applied, but now in a spatially selected slice. Using this spatial information, the fluid composition as well as the velocity can be determined as a function of height. An example of this data acquisition is shown in Figure 14.

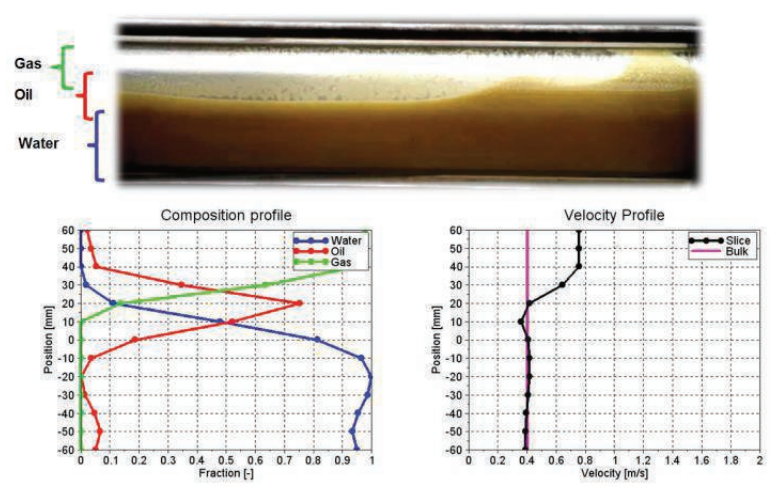


Figure 12 The lower left-hand figure shows the composition profile for the multiphase stratified wavy flow as shown in the upper figure. In the right-hand lower figure the corresponding time averaged velocity profile is depicted. (Gas: $5.8(\mathrm{a}) \mathrm{m}^{3} / \mathrm{h}$, Oil: $1.6 \mathrm{~m}^{3} / \mathrm{h}$, Water: $6.4 \mathrm{~m}^{3} / \mathrm{h}$, WLR: $80 \%$, GVF: $43 \%$ ).

\section{Design of the MR Multiphase Flow Meter}

The current flow meter is operated in a horizontal configuration. The design is an industrialized version of the improved and tested prototype version and has a full bore pipe. No specific upstream flow conditioning measures are required. The connecting flanges are made from stainless steel (SS) or carbon steel (CS). The overall flange-toflange length is about $3 \mathrm{~m}$. Maximum process temperature is $100^{\circ} \mathrm{C}$ and the minimum and maximum ambient operating temperatures range from $-40^{\circ} \mathrm{C}$ to $+65^{\circ} \mathrm{C}$. All electronics are mounted directly on the flow meter in two flame-proof boxes (Figure 13).

An important feature of the MR flow meter is that there are no protrusions into the pipe. Inside the flow meter, the transition is made from steel flanges to a glass fiber reinforced epoxy (GRE) pipe. A non-conductive pipe section is required to allow high frequency electromagnetic waves to be transmitted into the pipe. All measurements are made from outside of the tube. There is no feedthrough or anything else puncturing the tube.

The selected GRE pipe and flanges are frequently used in the oil and gas industry and possess all required certifications and approvals. The forces from the connected piping system are guided through the external housing in order to prevent mechanical loading of the GRE pipe.

The magnetic field strength in the main magnet is equal to the magnetic field strength in the pre-magnetization section. However, the magnetic field homogeneity of the main magnet is orders of magnitude higher than the homogeneity of the pre-magnetization section. The high field homogeneity is necessary for proper proton resonance measurements.

In contrast to the magnetic field strength inside the pipe, the magnetic field strength outside the meter housing is very weak $(<0.5 \mathrm{mT})$. This level is below the maximum field strength legally specified by standards. The value 0.5 $\mathrm{mT}$ is about 10 times stronger than the Earth's magnetic field strength.

In between the main magnet and the GRE pipe, the radio frequency coil is located. The RF coil is transmitting and receiving the signals and is matched and tuned to the Larmor precession of flowing phases by a matching and tuning circuit mounted in the electronics housing.

\section{Summary}

This paper provides an explanation of the physical concept of a magnetic resonance based multiphase flowmeter. Utilizing the concept of magnetic resonance imaging, it has - for the first time - been possible to directly quantify the flow of a free gas phase during multi-phase flow at industrial conditions.

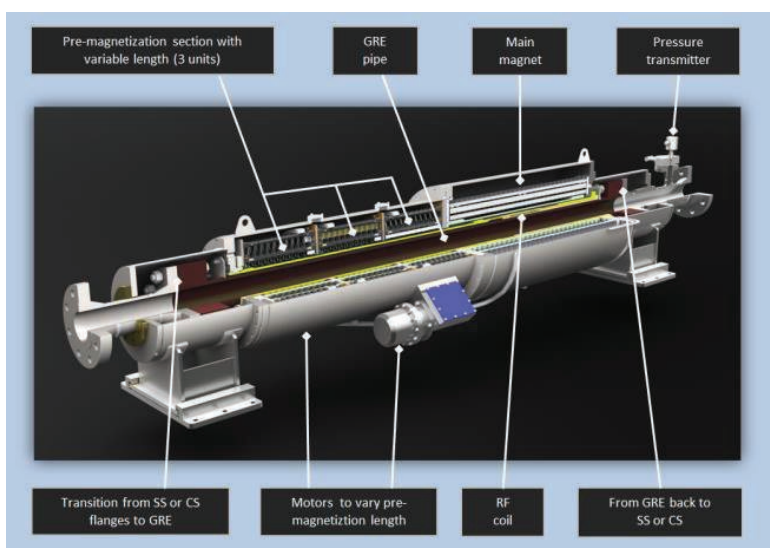

Figure 13 Mechanical design of the industrialized MR multiphase flow meter.

\section{Acknowledgement}

The authors of this paper like to express their appreciation to the entire development team for the tremendous amount of work that has been done throughout the last year. Further acknowledgement is made to the various operators of the flow facilities at which the meter has been tested.

\section{Bibliography}

[1] M. Appel and others, "Nuclear magnetic resonance and formation porosity," Petrophysics Houston, vol. 45, no. 3, pp. 296-307, 2004.

[2] J. Hogendoorn, A. Boer, M. Appel, H. de Jong, and R. de L. Shell, "Magnetic Resonance Technology A New Concept for Multiphase Flow Measurement," resonance, vol. 5, p. 6, 2013.

[3] Jankees Hogendoorn and et al., "Magnetic Resonance Multiphase Flowmeter: Measuring Principle and Broad Range Test Results," presented at the South East Asia Flow Measurement Conference, Kuala Lumpur, 2015.

[4] E. L. Hahn, "Spin echoes," Physical Review, vol. 80, no. 4 , p. $580,1950$.

[5] P. T. Callaghan, Principles of nuclear magnetic resonance microscopy, vol. 3. Clarendon Press Oxford, 1991.

[6] N. Bloembergen, E. M. Purcell, and R. V. Pound, "Relaxation Effects in Nuclear Magnetic Resonance Absorption," Phys. Rev., pp. 669-712, 1948.

[7] T. Osán, J. Ollé, M. Carpinella, L. Cerioni, D. Pusiol, M. Appel, J. Freeman, and I. Espejo, "Fast measurements of average flow velocity by Low-Field $1 \mathrm{H}$ NMR," Journal of Magnetic Resonance, vol. 209, no. 2, pp. 116-122, 2011. 